\title{
A new determination of the Pomeron intercept in hard processes
}

\author{
J. G. Contreras*, R. Peschanski ${ }^{\dagger}$ C. Royon ${ }^{\ddagger}$
}

\begin{abstract}
A method allowing for a direct comparison of data with theoretical predictions is proposed for forward jet production at HERA. It avoids the reconstruction of multi-parton contributions by expressing the experimental cuts directly as correction factors on the QCD forward jet crosssection. An application to the determination of the effective Pomeron intercept in the BFKL-LO parametrization from $d \sigma / d x$ data at HERA leads to a good fit with a significantly higher effective intercept, $\alpha_{P}=1.43 \pm 0.025$ (stat.) \pm 0.025 (syst.), than for proton (total and diffractive) structure functions. It is however less than the value of the pomeron intercept using dijets with large rapidity intervals obtained at Tevatron. We also evaluate the rapidity veto contribution to the higher order BFKL corrections. The method can be extended to other theoretical inputs.
\end{abstract}

\section{Introduction}

The study of forward jets at colliders is considered as the milestone of QCD studies at high energies, since it provides a direct way of testing the perturbative resummations of soft gluon radiation. More precisely, the study of one forward jet (w.r.t. the proton) in an electron-proton collider [1] seems to be a good candidate to test the energy dependence of hard QCD cross-sections. It is similar to the previous proposal of studying two jets separated by a large rapidity interval in hadronic colliders [2], for which only preliminary results are available [3]. This test is also possible in $\gamma^{*}-\gamma^{*}$ scattering [4] but here the statistics and the energy range are still insufficient to get a reliable determination of the physical parameters for hard QCD cross-sections. Indeed, the proposed (and favored for the moment being) set-up [1] is to consider jets with transverse momentum $k_{T}$ of the order of the photon virtuality $Q$ allowing to damp the QCD evolution as a function of $k_{T}$ (DGLAP evolution (5) in favor of the evolution in energy at fixed $k_{T}$ (BFKL evolution [6]).

Since proposal [1] was made, a set of interesting studies have been performed to check its relevance. On the experimental ground, H1 [7] and ZEUS [8 have published useful results with appropriate cuts (to be displayed later on) at relatively small $x$. On the theoretical ground, the general formulation and some quantitative estimates have been performed prior to experiments [9] confirming the interest in such processes. The recent theoretical analyses have been mainly based on the use of Monte-Carlo simulations, including the multi-parton cross-sections and starting from the various frameworks in competition [10]. Quite a few analyses arrive at a satisfactory description of the data, taking into account the specific parametrizations which are choosen. Indeed, the BFKL-based Monte-Carlo 10 lead to quite satisfactory results, while those based on DGLAP evolution meet some difficulty to describe the datat.

However, there still remains a problem in the interpretation of those results. Due to the difficulty in handling the experimental cuts without introducing in the simulation the whole set of theoretical n-parton contributions to the cross-section, it appears difficult to avoid the uncertainties of the reconstruction (with the parameters and constraints which are needed to define the scheme in practice). It seems thus difficult to determine unambiguously genuine theoretical parameters defining the cross-section one is looking at. One example is the dependence in parameters such as infra-red cut-offs, which are not a-priori required in the expression of the total $d \sigma / d x$ jet cross sections. Another illustration is the so-called "consistency constraint" which appears very useful [11] in the expression of next-to-leading corrections to the BFKL formula coming from the n-parton contributions, but again is not expressed in terms of the $d \sigma / d x$ jet cross sections itself. In fact, it does not seem easy, in those schemes, to extract with some precision the value of the effective Pomeron intercept $\alpha_{P}$, i.e. the main theoretical parameter describing the theoretical energy dependence in this process. As we know, this parameter is of primordial importance to evaluate the amount of next-leading corrections in a BFKL framework [12] and to confront its effective value with the recent theoretical determinations 13 .

We want to address this problem in a quite different way, that is on focussing on the jet cross section $d \sigma / d x$ observable itself, by a consistent treatment of the experimental cuts and minimizing the uncertainties for that particular observable. Let us remark that our approach is not intended to provide a substitution to the other methods, since

\footnotetext{
*Departamento de Física Aplicada, CINVESTAV-IPN, Unidad Mérida, A. P. 73 Cordemex, 97310 Mérida, Yucatán, México ${ }^{\dagger}$ CEA, Service de Physique Theorique, CE-Saclay, F-91191 Gif-sur-Yvette Cedex, France

${ }^{\ddagger}$ Service de Physique des Particules, CE-Saclay, F-91191 Gif-sur-Yvette Cedex, France

${ }^{1}$ Note however, that some more refined versions of DGLAP evolution including contributions from the resolved off-mass-shell photon can describe the data 10 .
} 
the Monte-Carlo simulations have the great merit of making a set of predictions for various observables. Hence, our method has to be considered as complementary to the others and dedicated to a better determination of the effective Pomeron intercept using the $d \sigma / d x$ data. As we shall see, it will fix more precisely this parameter, but it will leave less constrained other interesting parameters, such as the cross-section normalization.

One fruitful outcome of the method proposed in the present paper is the possibility of comparing the effective intercept with its determination in other processes involving QCD at high energy. In fact, using the parameters determined from forward jets at HERA, it is possible to compare with double jet production at Tevatron following Ref. [2] for which preliminary experimental analyses have been performed [3] and find a high value of the intercept $\left(\alpha_{P}=1.7 \pm .1 \pm .1\right.$ in Ref. [3] $)$. It can also be confronted with the effective BFKL analysis of proton structure functions at small- $x_{B j}$, which give rather low values ${ }^{2}\left(\alpha_{P} \sim 1.1-1.21\right.$ see Ref. 14] $)$. However, in those cases, the result may be different, since non-perturbative effects related to the "soft" proton scales are expected to influence the determination of parameters.

Thus, the comparison of the effective BFKL parameter $\alpha_{P}$ obtained for the forward jet production cross-section allows for a study of QCD at high energy, aiming at a better understanding of the corrections to the leading-order BFKL predictions [12, 11].

The plan of our study is the following: in section 2, we introduce the QCD formalism and our method for determining $\alpha_{P}$. In the following section $\mathbf{3}$ we determine the kinematic correction factors to the forward jet cross-section data on $d \sigma / d x$ due to the experimental cuts. In the following section 4, we perform and discuss a (separately and then common) fit to H1 and ZEUS data. This determines the BFKL parameter $\alpha_{P}$ which is subsequently used in section 4 for a comparison with the two-jet cross-section at Tevatron from the experimental D0 analysis. Discussions on these results and comparison with the BFKL study of (total and diffractive) structure functions are presented in section $\mathbf{5}$ and conclusions and outlook in section 6.

\section{Formalism}

The cross-section for forward jet production at HERA in the dipole model reads [9]:

$$
\begin{gathered}
\frac{d^{(4)} \sigma}{d x d Q^{2} d x_{J} d k_{T}^{2} d \Phi}=\frac{\pi N_{C} \alpha^{2} \alpha_{S}\left(k_{T}^{2}\right)}{Q^{4} k_{T}^{2}} f_{e f f}\left(x, \mu_{f}^{2}\right) \Sigma e_{Q}^{2} \int_{\frac{1}{2}-i \infty}^{\frac{1}{2}+i \infty} \frac{d \gamma}{2 i \pi}\left(\frac{Q^{2}}{k_{T}^{2}}\right)^{\gamma} \times \\
\times \exp \{\epsilon(\gamma, 0) Y\}\left[\frac{h_{T}(\gamma)+h_{L}(\gamma)}{\gamma}(1-y)+\frac{h_{T}(\gamma)}{\gamma} \frac{y^{2}}{2}\right]-\exp \{\epsilon(\gamma, 1) Y\} \cos 2 \Phi\left[\frac{h_{T}(\gamma)}{\gamma} \frac{\gamma(1-\gamma)}{(\gamma+1)(2-\gamma)}\right]
\end{gathered}
$$

where

$$
\begin{aligned}
Y & =\ln \frac{x_{J}}{x} \\
\epsilon(\gamma, p) & =\bar{\alpha}[2 \psi(1)-\psi(p+1-\gamma)-\psi(p+\gamma)] \\
f_{e f f}\left(x, \mu_{f}^{2}\right) & =G\left(x, \mu_{f}^{2}\right)+\frac{4}{9} \Sigma\left(Q_{f}+\bar{Q}_{f}\right) \\
\mu_{f}^{2} & \sim k_{T}^{2},
\end{aligned}
$$

are, respectively, $Y$ the rapidity interval between the photon probe and the jet, $\epsilon(\gamma, p)$ the BFKL kernel eigenvalues, $f_{\text {eff }}$ the effective structure function combination, and $\mu_{f}$ the corresponding factorization scale. The main BFKL parameter is $\bar{\alpha}$, which is the (fixed) value of the effective strong coupling constant in LO-BFKL formulae. Note that we gave for completion the full BFKL formula including the azimuthal dependence but we will stick to the azimuth-independent contribution with the dominant $\exp \{\epsilon(\gamma, 0) Y\}$ factor.

The so-called "impact factors"

$$
\left(\begin{array}{c}
h_{T} \\
h_{L}
\end{array}\right)=\frac{\alpha_{S}\left(k_{T}^{2}\right)}{3 \pi \gamma} \frac{(\Gamma(1-\gamma) \Gamma(1+\gamma))^{3}}{\Gamma(2-2 \gamma) \Gamma(2+2 \gamma)} \frac{1}{1-\frac{2}{3} \gamma}\left(\begin{array}{c}
(1+\gamma)\left(1-\frac{\gamma}{2}\right) \\
\gamma(1-\gamma)
\end{array}\right)
$$

are obtained from the $k_{T}$ factorization properties [17] of the coupling of the BFKL amplitudes to external hard probes. The same factors can be related to the photon wave functions 18, 16] within the equivalent context of the QCD dipole model [19].

\footnotetext{
${ }^{2}$ Note however that taking into account the full BFKL formula may lead to higher $\alpha_{P}$, namely $1.2-1.3$, see Ref. [15], for total structure functions and even reach .4, see Ref. [16], for diffractive proton structure functions.
} 
Our goal is to compare as directly as possible the theoretical parametrization (1) to the data which are collected in experiments [7,8]. The crucial point is how to take into account the experimentally defined kinematic cuts listed in Table I for the reported three sets of data (two for $\mathrm{H} 1$ with $k_{T}=3.5$ or $5 \mathrm{GeV}$, and one for ZEUS).

\begin{tabular}{|c|c|}
\hline H1 cuts & ZEUS cuts \\
\hline \hline$E_{e}^{\prime}>11 \mathrm{GeV}$ & $E_{e}^{\prime}>10 \mathrm{GeV}$ \\
$160 \leq \theta_{e}^{\prime} \leq 173 \mathrm{deg}$. & \\
$y>0.1$ & $y>0.1$ \\
$7 \leq \theta_{\text {jet }} \leq 20 \mathrm{deg}$. & $\theta_{\text {jet }} \geq 8.5 \mathrm{deg}$. \\
$k_{T j e t} \geq 3.5$ or $5 \mathrm{GeV}$ & $k_{\text {Tjet }} \geq 5 \mathrm{GeV}$ \\
$x_{\text {jet }}>0.035$ & $x_{\text {jet }}>0.036$ \\
$0.5<\frac{k_{T}^{2}}{Q^{2}}<2$ & $0.5<\frac{k_{T}^{2}}{Q^{2}}<2$ \\
$10^{-4}<x<4.10^{-3}$ & $4.510^{-4}<x<4.510^{-2}$ \\
\hline
\end{tabular}

Table I- Experimental cuts (H1/ZEUS)

The main problem to solve is to investigate the effect of these cuts on the determination of the integration variables leading to a prediction for $d \sigma / d x$ from the given theoretical formula for $d^{(4)} \sigma$ as given in formula (1). The effect is expected to appear as bin-per-bin correction factors to be multiplied to the theoretical cross-sections for average values of the kinematic variables for a given $x$-bin before comparing to data (e.g. fitting the cross-sections).

The idea of our method is threefold: i) for each $x$-bin, determining the average values of $x, Q^{2}, E_{J}, k_{T}$ from a known and reliable Monte-Carlo simulation of the cross-sections. For this sake, we use the Ariadne Monte-Carlo programme [21]; ii) choosing a set of integration variables over $d^{(4)} \sigma$ in (11) in such a way to match closely the experimental cuts and minimize the variation of the cross-sections over the bin size; iii) fixing the correction factors due to the experimental cuts for each $x$-bin, by a random simulation of the kinematic constraints with no dynamical input.

The point i) proposed already in 20] allows a determination of which average values of the kinematic variables have to be taken in the theoretical formula (11) for each experimental $x$-bin. The point ii) comes from the crucial requirement to minimize the variation (over the $x$-bin) of the variables to be retained for the integration. Indeed, since the integration procedure multiplies the central value of the integrand by the size of the integration bins, it is compulsory to choose adequate variables which lead to a smooth dependence of the integrand and of the effect of the kinematic cuts.

This double stringent requirement can be solved for the forward jet $d \sigma / d x$. For this sake we choose

$$
\frac{d \sigma}{d x}=\int\left[Q^{6} \frac{d^{(4)} \sigma}{d x d Q^{2} d x_{J} d k_{T}^{2} d \Phi}\right] \times \Delta\left(\frac{1}{Q^{2}}\right) \Delta x_{J} \Delta\left(\frac{k_{T}^{2}}{Q^{2}}\right) \Delta \Phi .
$$

The property of this non-trivial choice is the following. The integration variables are choosen in such a way that the expression in the square brackets $\left[Q^{6} \ldots\right]$ in $(7)$ is dependent on the ratio $\frac{k_{T}^{2}}{Q^{2}}$ and not on each scale separately. Looking at the experimental cuts (see Table I), it becomes clear that the choice of this scale-invariant integrand minimizes the variation of the observable on the bin, while each scale $k_{T}^{2}$ and $Q^{2}$ presents large variations and thus would generate large integration errors. Indeed, various numerical studies we have performed have demonstrated that it was a sine qua non stability condition for the fits. The overall azimuthal integration $(\Delta \Phi=2 \pi)$ cancels the second term in (1).

\section{Correction Factors}

The experimental correction factors have been determined using a toy Monte-Carlo designed as follows. We generate flat distributions in the variables $k_{T}^{2} / Q^{2}, 1 / Q^{2}, x_{J}$, using reference intervals which include the whole of the experimental phase-space (the $\Phi$ variable is not used in the generation since all the cross-section measurements are $\phi$ independent). In practice, we get the correction factors by counting the numbers of events which fulfill the experimental cuts given in Table $\mathbf{I}$ for each $x$-bin. The correction factor is obtained by the ratio to the number of events which pass the experimental cuts and the kinematic constraints, and the number of events which fullfil only the kinematic constraints,i.e. the so-called reference bin. 


\begin{tabular}{|c|c|c|c|c|c|}
\hline $\mathrm{x}$ & $\sigma$ & $Q^{2}$ & $E_{\text {jet }}$ & $k_{T}$ & Corr. Factor $\left(.10^{-3}\right)$ \\
\hline \hline 0.00036 & 202.5 & 13.9 & 32.6 & 4.5 & 0.270 \\
0.00073 & 342. & 21.5 & 34.4 & 5.0 & 0.993 \\
0.0012 & 224. & 26.9 & 36.9 & 5.5 & 1.14 \\
0.0017 & 138. & 31.4 & 38.1 & 5.8 & 1.11 \\
0.0024 & 67. & 38.1 & 38.8 & 6.3 & 0.921 \\
0.0035 & 32. & 47.0 & 37.9 & 6.9 & 0.711 \\
\hline \hline
\end{tabular}

Table IIa- Average values of kinematic quantities and correction factors - $\mathrm{H} 1 k_{T}>3.5 \mathrm{GeV}$

\begin{tabular}{|c|c|c|c|c|c|}
\hline $\mathrm{x}$ & $\sigma$ & $Q^{2}$ & $E_{j e t}$ & $k_{T}$ & Corr. Factor $\left(.10^{-3}\right)$ \\
\hline \hline 0.00036 & 27.5 & 18.0 & 35.9 & 5.5 & 0.108 \\
0.00073 & 126. & 27.0 & 36.5 & 5.8 & $0.695^{*}$ \\
0.0012 & 132. & 32.2 & 37.9 & 6.3 & 0.895 \\
0.0017 & 96. & 34.8 & 39.3 & 6.5 & 0.979 \\
0.0024 & 55. & 40.1 & 39.4 & 6.7 & 0.870 \\
0.0035 & 28. & 48.2 & 39.6 & 7.2 & 0.696 \\
\hline \hline
\end{tabular}

Table IIb- Average values of kinematic quantities and correction factors - $\mathrm{H} 1 k_{T}>5 G e V$

\begin{tabular}{|c|c|c|c|c|c|}
\hline $\mathrm{x}$ & $\sigma$ & $Q^{2}$ & $E_{\text {jet }}$ & $k_{T}$ & Corr. Factor $\left(.10^{-3}\right)$ \\
\hline \hline 0.0006 & 114.0 & 28.0 & 36.5 & 6.3 & $0.304^{*}$ \\
0.0011 & 96.2 & 39.0 & 38.0 & 6.9 & 0.656 \\
0.0019 & 77.8 & 50.7 & 39.9 & 7.6 & 0.966 \\
0.0033 & 34.4 & 75.6 & 43.8 & 8.7 & 0.996 \\
0.006 & 14.1 & 113.6 & 49.6 & 10.4 & 0.995 \\
0.01 & 6.53 & 176.4 & 58.5 & 12.9 & $0.896^{*}$ \\
0.018 & 2.65 & 244.7 & 67.3 & 15.1 & $0.653^{*}$ \\
0.031 & 0.65 & 366.8 & 78.8 & 18.8 & $0.373^{*}$ \\
\hline \hline
\end{tabular}

Table IIc- Average values of kinematic quantities and correction factors - ZEUS $k_{T}>5 G e V$

The correction factors are given in Table IIa for H1 $\left(k_{T}>3.5 \mathrm{GeV}\right)$, Table IIb for H1 $\left(k_{T}>5 \mathrm{GeV}\right)$, and Table IIc for ZEUS bins together with the value of the bin centers determined with the full Monte-Carlo simulation [21], and the experimental values of the cross-sections. We note that the correction factors are quite different from one $x$-bin to an other and much less than one (in $10^{-3}$ units), explicitely showing that the experimental cuts play an important role in the cross-section measurement, and that these factors are compulsory to be taken into account if we want to get a direct comparison with the theoretical cross-sections. We also note that the correction factors are very much different from one another at very low $x$, showing that the acceptance of these bins is quite low. This is also why it is not so easy to be able to get a correct value of the measured cross-section after cuts in those bins. We also get the same order of magnitude for the correction factors for the H1 and ZEUS experiments because the experimental cuts

\footnotetext{
${ }^{3}$ Note that we did not use the full Monte-Carlo to get the correction factors in order to avoid any strong model dependence as these factors are only due to kinematic effects. It is however more difficult to use a toy Monte Carlo to get accurate values for the bin centers, and this is why we used a full Monte Carlo for this sake. However, the dependence of the theoretical cross-section on the bin centers is minimized by our specific choice of kinematic variables (see formula (7)).
} 
are quite similar. The differences between both experiments are due mainly to the fact that the range in $x$ and $Q^{2}$ is much lower for $\mathrm{H} 1$ than for ZEUS (the reference bin for $\mathrm{H} 1$ goes to lower $Q^{2}$ compared to ZEUS).

\section{Fits}

Using the kinematic correction factors determined as described in the previous section, we perform a fit to the H1 and ZEUS data with only two free parameters. these are the effective strong coupling constant in LO BFKL formulae $\bar{\alpha}$ corresponding to the effective Lipatov intercept $\alpha_{P}=1+4 \log 2 \bar{\alpha} N_{C} / \pi$, and the cross-section normalisation. The obtained values of the parameters and the $\chi^{2}$ of the fit are given in Table III for a fit to the H1 and ZEUS data separately, and then to the H1 + ZEUS data together. Note that one $\mathrm{H} 1$ point at $k_{T}>5 \mathrm{GeV}\left(7.310^{-4}\right)$, and four ZEUS points $\left(x=4.10^{-4}\right.$, and the three highest-x points), were not taken into account in the fit and are distinguished in Tables II with a star. We will discuss this selection in a little while.

\begin{tabular}{|c|c|c|c|c|}
\hline fit & $\bar{\alpha}$ & $\alpha_{P}$ & Norm. & $\chi^{2}(/ d o f)$ \\
\hline \hline H1 & $0.17 \pm 0.02 \pm 0.01$ & $1.44 \pm 0.05 \pm 0.025$ & $29.4 \pm 4.8 \pm 5.2$ & $5.7(/ 9)$ \\
ZEUS & $0.20 \pm 0.02 \pm 0.01$ & $1.52 \pm 0.05 \pm 0.025$ & $26.4 \pm 3.9 \pm 4.7$ & $2.0(/ 2)$ \\
H1+ZEUS & $0.16 \pm 0.01 \pm 0.01$ & $1.43 \pm 0.025 \pm 0.025$ & $30.7 \pm 2.9 \pm 3.5$ & $12.0(/ 13)$ \\
\hline
\end{tabular}

Table III- Fit results

The $\chi^{2}$ of the fits have been calculated using statistical error only and are very satisfactory (about 0.6 per point for H1 data, and 1. per point for ZEUS data). We give both statistical and systematic errors for the fit parameters. The values of the Lipatov intercept are close to one another and compatible within errors for the H1 and ZEUS sets of data, and indicate a preferable medium value $\left(\alpha_{P}=1.4-1.5\right)$. We also notice that the ZEUS data have the tendency to favour a higher exponent, but the number of data points used in the fit is much smaller than for H1, and the H1 data are also at lower $x$. The normalisation is also compatible between ZEUS and H1. The fit results are shown in Figure 1 and compared with the H1 and ZEUS measurements.

Let us discuss our selection criterium for the fits. Both lowest $x$ points for H1 and ZEUS show large correction factors but only the lowest $x$ point for ZEUS lies a bit above the prediction, which shows the relevance of the correction factors we determined. On the other hand, the three highest $x$ points for ZEUS cannot be described by a BFKL fit probably because the $x$-value is too high $\left(x>10^{-2}\right)$. Consider now the second lower $x$ point at $k_{T}>5 \mathrm{GeV}$ for the $\mathrm{H} 1$ experiment that we suppressed from the fit (see Table $\mathbf{I I b}$ ). If we include it in the fit the $\chi^{2}$ value goes from 5.7 to 32 , which is due to the small statistical error of this data point (the systematic error is on the contrary very large). By comparison, including the lowest $\mathrm{x}$ point for ZEUS changes the $\chi^{2}$ from 2.0 to 7.9. In the same way, including the highest $\mathrm{x}$ points still increases the $\chi^{2}$ to 67.4 , showing clearly that these highest $\mathrm{x}$ points cannot be described using the BFKL formalism. It is interesting to note that all similar discrepancies appear also in other types of fitting procedures, e.g. in Ref. [22].

\section{Comparison with other processes}

The final result of our new determination of the effective pomeron intercept is $\alpha_{P}=1.43 \pm 0.025$ (stat.) \pm 0.025 (syst.). This high value of the intercept leads to the following remarks. Our analysis confirms the trend observed using DGLAP based Monte-Carlo [10] which have difficulties to reproduce the forward jet cross-section due to a low effective pomeron intercept when both $k_{T}^{2}$ and $Q^{2}$ scales are of the same order.

On the other hand, our method allows a direct comparison of the intercept values with those obtained in other experimental processes, i.e. $\gamma^{*} \gamma^{*}$ cross-sections at LEP [ [ [], jet-jet cross-sections at Tevatron at large rapidity intervals [3], $F_{2}$ and $F_{2}^{D}$ proton structure function measurements [14 [16]. Let us first consider the known determinations of the effective intercepts in $F_{2}$ and $F_{2}^{D}$ measurements at HERA [23]. It is known that the effective intercept determined in these measurements is rather low $(1.2-1.3)$. This is the reason why these data can be both described by a DGLAP or a BFKL-LO fit 5 .

\footnotetext{
${ }^{4}$ It is interesting to note that the "hard" Pomeron intercept obtained within the framework of two-Pomeron models 24 fits with our determination. However our parametrization (11) corresponds to only one Pomeron.

${ }^{5}$ Note that in the BFKL descriptions of these data 15, 16, the effective intercept is taken to be constant, while the $Q^{2}$ dependence comes from the BFKL integration (see for instance formula (䜣)
} 

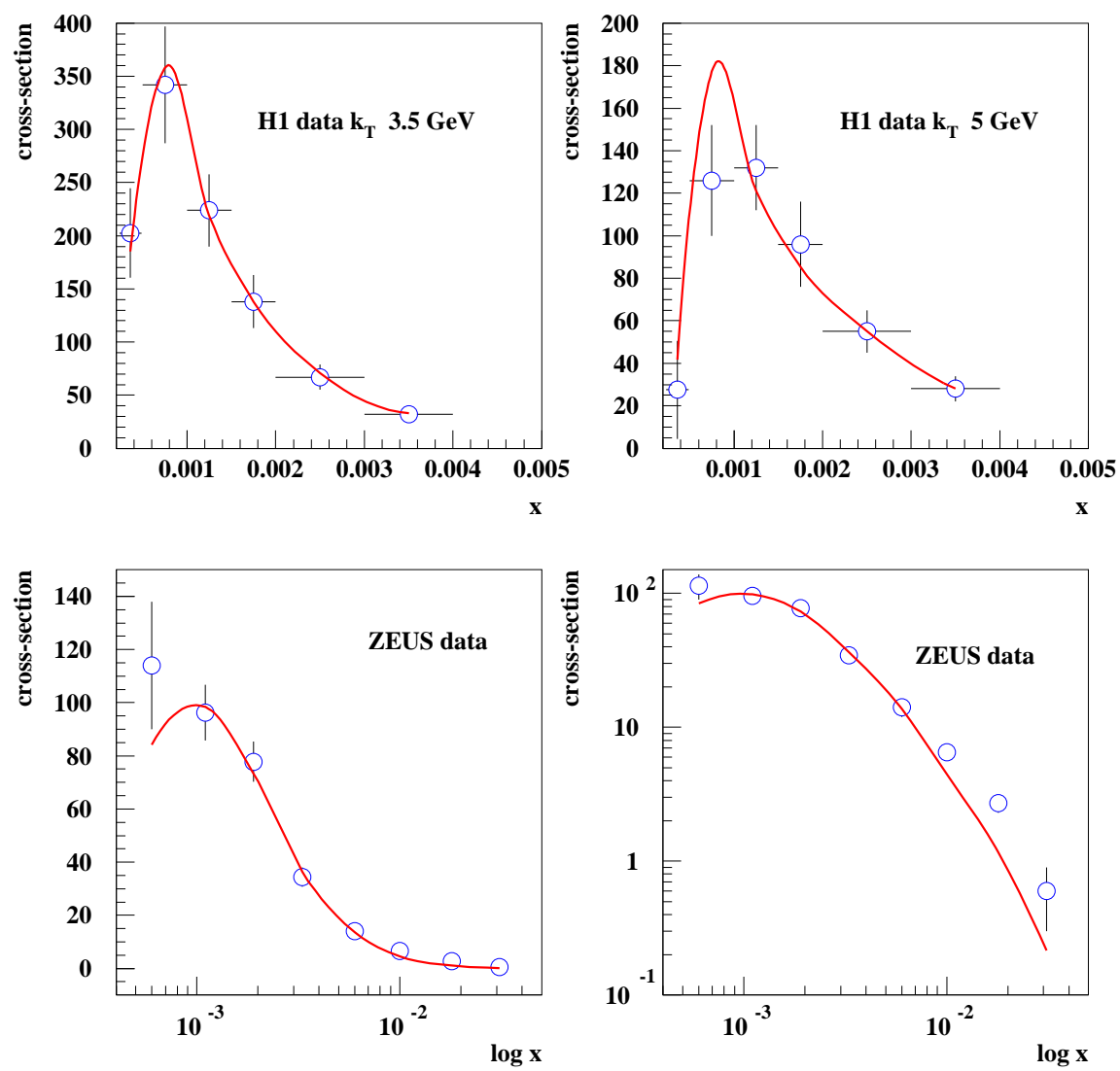

FIG. 1. The H1 data $\left(k_{T}>3.5 \mathrm{GeV}, k_{T}>5 \mathrm{GeV}\right)$, and the ZEUS data are compared with the result of the fit. ZEUS data are also displayed in logarithmic scales in vertical coordinates to show the discrepancy at high $x$ values.

Now let us consider processes initiated by two hard probes which allow a more direct comparison between experiments and BFKL predictions. These processes suppress DGLAP evolution by selecting events with comparable hard scales for both hard probes. Recent data on $\gamma^{*} \gamma^{*}$ cross-section measurements at LEP [25] lead to a BFKL description with a low effective intercept compatible with the one of $F_{2}$ and $F_{2}^{D}$ at HERA $\left(\alpha_{P}=1.2-1.3\right.$ 肺) . The fact that similar values of the intercepts are found could be interpreted by sizeable higher order corrections to BFKL equation. On the other hand, it is interesting to note that our result based on forward jet measurement at HERA obtained in comparable $Q^{2}\left(Q^{2} \sim 10 \mathrm{GeV}^{2}\right)$ and rapidity $(Y \sim 3-4)$ domains is quite different. The value of the intercept is significantly higher.

It is also fruitful to compare our results with the effective intercept we obtain from recent preliminary dijet data obtained by the D0 Collaboration at Tevatron [3]. The measurement consists in the ratio $R=\sigma_{1800} / \sigma_{630}$ where $\sigma$ is the dijet cross-section at large rapidity interval $Y \sim \Delta \eta$ for two center-of-mass energies $(630$ and $1800 \mathrm{GeV}), \Delta \eta_{1800}=4.6$, $\Delta \eta_{630}=2.4$. The experimental measurement is $R=2.9 \pm 0.3$ (stat.) \pm 0.3 (syst.). Using the Mueller-Navelet formula [2], this measurement allows us to get a value of the effective intercept for this process $\square$

\footnotetext{
${ }^{6}$ The statistics for these data is still very low. L3 and OPAL Collaborations have released the cuts used to enhance BFKL effects to get more statistics [25:4. These data can be both described by BFKL and DGLAP evolution equations.

${ }^{7}$ Formula (8) is obtained after integration over the jet tranverse energies at 630 and $1800 \mathrm{GeV}, E_{T_{1}}, E_{T_{2}}$. We note that a non integrated formula shows a sizeable dependence on $E_{T_{1}} / E_{T_{2}}$, which could be confronted with experiment [26].
} 


$$
R=\frac{\int_{\frac{1}{2}-i \infty}^{\frac{1}{2}+i \infty} \frac{d \gamma}{2 i \pi \gamma(1-\gamma)} e^{\epsilon(\gamma, 0) \Delta \eta_{1800}}}{\int_{\frac{1}{2}-i \infty}^{\frac{1}{2}+i \infty} \frac{d \gamma}{2 i \pi \gamma(1-\gamma)} e^{\epsilon(\gamma, 0) \Delta \eta_{630}}}
$$

We get $\alpha_{P}=1.65 \pm 0.05$ (stat.) \pm 0.05 (syst.), in agreement with the value obtained by D0 using a saddle-point approximation [3]. This intercept is higher than the one obtained in the forward jet study.

The question arises to interpret the different values of the effective intercept. It could reasonably come from the differences in higher order QCD corrections for the BFKL kernel and/or in the impact factors depending on the initial probes ( $\gamma^{*}$ vs. jets). In order to evaluate the approximate size of the higher order BFKL corrections, we will use their description in terms of rapidity veto effects [27]. In formula (11), we make the following replacement

$$
\exp (\epsilon(\gamma, 0) Y) \rightarrow \Sigma_{n=0}^{\infty} \theta(Y-(n+1) b) \frac{[\epsilon(\gamma, 0)(Y-(n+1) b)]^{n}}{\Gamma(n+1)} .
$$

The Heaviside function $\theta$ ensures that a BFKL ladder of $n$ gluons occupies $(n+1) b$ rapidity interval where $b$ parametrises the strength of NLO BFKL corrections. The value of the leading order intercept is fixed to $\alpha_{p}=1.75\left(\alpha_{S}\left(Q^{2}=10\right)=0.28\right)$, where $Q^{2}=10 \mathrm{GeV}^{2}$ is inside the average range of $Q^{2}$ in the forward jet measurement. The fitted value of the $b$ parameter obtained using the forward jet data is found to be $1.28 \pm 0.08$ (stat.) \pm 0.02 (syst.). Imposing the same value of $\alpha_{P}$ with Tevatron data gives $b=0.21 \pm 0.11$ (stat.) \pm 0.11 (syst.). Note that the theoretical value of $b$ for the NLO BFKL kernel is expected to be of the order 2.4, which is also compatible with the result obtained for the $\gamma^{*} \gamma^{*}$ cross-section. A contribution from the NLO impact factors is not yet known, and could perhaps explain the different values of $b$.

\section{Conclusions}

To summarize our results, using a new method to disantangle the effects of the kinematic cuts from the genuine dynamical values of the forward jet cross-sections at HERA, we find that the effective pomeron intercept is $\alpha_{P}=$ $1.43 \pm 0.025$ (stat.) \pm 0.025 (syst.). It is much higher than the soft pomeron intercept, and, among those determined in hard processes, it is intermediate between $\gamma^{*} \gamma^{*}$ interactions at LEP and dijet productions with large rapidity intervals at Tevatron.

Looking for an interpretation of our results in terms of higher order BFKL corrections expressed by rapidity gap vetoes $b$ between emitted gluons, we find a value of $b=1.3$, which is sizeable but less than the theoretically predicted [12] value for the NLO BFKL kernel $(b=2.4)$. The observed dependence in the process deserves further more precise studies 28].

Last but not least, the derivation of the correction factors given in Table II is independent of the theoretical input and could be used to test any model suitable for the jet cross-section.

\section{Acknowledgments}

We would like to thank Lev Lipatov for his fruitful remarks and suggestions. One of us (J.G.C.) acknowledges supports by CONACyT.

[1] A.H.Mueller, Nucl. Phys. B (Proc. Suppl.) 18C (1991) 125.

[2] A.H.Mueller and H.Navelet, Nucl. Phys. B282 (1987) 107.

[3] A.Goussiou, for the D0 collaboration, Dijet Cross section at large $s / Q^{2}$ in $\bar{p} p$ Collisions, presented at the 'International Europhysics Conference On High-Energy Physics' (EPS-HEP 99), Tampere, Finland, July, 1999.

[4] S.Brodsky, V.S.Fadin, V.T.Kim, L.N.Lipatov, G.B.Pivovarov, JETP Lett. 70 (1999) 155 , M.Boonekamp. A.De Roeck, C.Royon, S.Wallon, Nucl.Phys. B555 (1999) 540, for a recent review and references, Ch. Royon, BFKL signatures at a linear collider, invited talk given at the International Workshop on Linear Colliders (LCWS99), April 28- May 5, Sitges (Spain), hep-ph/9909295.

[5] G.Altarelli and G.Parisi, Nucl. Phys. B126 18C (1977) 298. V.N.Gribov and L.N.Lipatov, Sov. Journ. Nucl. Phys. (1972) 438 and 675. Yu.L.Dokshitzer, Sov. Phys. JETP. 46 (1977) 641. 
[6] L.N.Lipatov, Sov. J. Nucl. Phys. 23 (1976) 642; V.S.Fadin, E.A.Kuraev and L.N.Lipatov, Phys. lett. B60 (1975) 50; E.A.Kuraev, L.N.Lipatov and V.S.Fadin, Sov.Phys.JETP 44 (1976) 45, 45 (1977) 199; I.I.Balitsky and L.N.Lipatov, Sov.J.Nucl.Phys. 28 (1978) 822.

[7] H1 Collaboration, C.Adloff et al. Nucl. Phys. B538 (1999) 3.

[8] ZEUS Collaboration, J.Breitweg et al. Eur. Phys. J. C6 (1999) 239.

[9] J.Bartels, A.De Roeck, M.Loewe, Zeit. für Phys. C54 (1992) 921; W-K. Tang, Phys. lett. B278 (1992) 635; J.Kwiecinski, A.D.Martin, P.J.Sutton, Phys.Rev. D46 (1992) 921.

[10] See, e.g. J.Bartels, V.Del Duca, A.De Roeck, D.Graudenz, M.Wüsthoff, Phys. lett. B384 (1996) 300, J.Kwiecinski, A.D.Martin, J.J.Outhwaite, Eur. Phys. J. C9 (1999) 611, for the BFKL approach. For different approches and recent review and references, B.Pötter, forward Jet Production at HERA, hep-ph/9909320.

[11] J.Kwiecinski, A.D.Martin, P.J.Sutton, Zeit. für Phys. C71 (1996) 585.

[12] V.S. Fadin and L.N. Lipatov Phys. Lett. B429 (1998)127. M. Ciafaloni Phys. Lett. B429 (1998) 363. M. Ciafaloni and G. Camici Phys. Lett. B430 (1998) 349.

[13] See, for a recent review and references, C.R. Schmidt, status of the BFKL resummation program, to be published in the proceedings of American Physical Society (APS) Meeting of the Division of Particles and Fields (DPF 99), Los Angeles, CA, 5-9 Jan 1999, hep-ph/9904368.

[14] H.Abramowicz, Diffraction and the Pomeron Contribution to the 19th International Symposium on Lepton and Photon Interactions at High-Energies (LP 99), Stanford, California, 9-14 Aug 1999, hep-ph/0001054.

[15] H. Navelet, R. Peschanski, Ch. Royon, Phys. Lett. B366 (1995) 329. H. Navelet, R. Peschanski, Ch. Royon, S. Wallon, Phys. Lett. B385 (1996) 357.

[16] S.Munier, R.Peschanski, Ch.Royon, Nucl.Phys. B534 (1998) 297.

[17] S. Catani, M. Ciafaloni, F. Hautmann, Nucl. Phys. B366 (1991) 135. J. C. Collins, R. K. Ellis, Nucl. Phys. B360 (1991) 3. E. M. Levin, M. G. Ryskin, Yu. M. Shabelskii, A. G. Shuvaev, Sov. J. Nucl. Phys. 53 (1991) 657.

[18] J.D.Bjorken, J.Kogut and Soper, Phys.Rev. D3 (1971) 1382. N.N.Nikolaev, B.G.Zakharov, Zeit. für. Phys. C49 (1991) 607; Phys. Lett. B332 (1994) 184.

[19] A.H.Mueller, Nucl. Phys. B415 (1994) 373; A.H.Mueller and B.Patel, Nucl. Phys. B425 (1994) 471; A.H.Mueller, Nucl. Phys. B437 (1995) 107.

[20] J.G. Contreras Phys. Lett. B446 (1999) 158.

[21] L.Lönnblad, Comp. Phys. Comm. 71 (1992) 15.

[22] J.Kwiecinski, A.D.Martin, J.J.Outhwaite, see ( 10$]$ ).

[23] H1 coll., Nucl.Phys. B470 (1996) 3, ZEUS Coll., Z.Phys. C72 (1996) 399, H1 coll., Z.Phys. C76 (1997) 613, ZEUS coll., Eur. Phys. J. C1 (1998) 81.

[24] A. Donnachie, P.V. Landshoff Phys.Lett. B437 (1998) 408, B470 (1999) 243, and hep-ph/9912312.

[25] L3 Coll., Phys. Lett. B 453 (1999) 94, M.Przybycien, OPAL Coll., contribution to the PHOTON99 conference, Freiburg, Germany, 23-27 May 1999.

[26] R.Peschanski, C.Royon, proceedings of the workshop on physics at Tevatron Run II, to be published.

[27] L.N.Lipatov, talk presented at the 4th Workshop on Small-x and Diffractive Physics, FNAL, September 1998, C.Schmidt, Phys.Rev. D60 (1999) 074003, J.Forshaw, D.A.Ross, A.Sabio Vera, Phys.Lett. B455 (1999) 273-282.

[28] V.T.Kim, L.N.Lipatov, R.Peschanski, G.Pivovarov, C.Royon, in progress. 